\title{
Combined Balanced Ternary Number System: An Approach to a New Computational Number System combining The Ternary Number System and the Balanced Ternary Number System in the field of Computational Mathematics
}

\author{
Md. Masudur Rahman \\ Noakhali Science and Technology University \\ Bangladesh
}

\author{
Md. Tanzil Mehadi Bappy \\ Noakhali Science and Technology University \\ Bangladesh
}

\begin{abstract}
Logical systems are the core essence of our current computation system and these logics are based on binary number system. For the last few decades binary has been used as a core system. But in the upcoming future, it would require more efficient performance, high rate of computation and strong base in machine control system and artificial intelligence. So, the upgrade of computer number system is very necessary for more scope, speed, computation and analysis. The next step to move from binary is ternary (more precisely balanced ternary) and it is already used to develop a system several times. The implementable ternary number system in computer is the balanced ternary number system which was previously used to develop "SETUN". Actually, the balanced ternary number system is the binary implemented form of ternary using the system of overflow throughout the entire conversion. This is a continuous overflow system and overlaps the value every single time when the remainder is two (2). In order to overlap the value it is necessary to check if the remainder is 2 or not; this check increases the number of logical condition used during the conversion. Thus continuous overflow generally implies in complexity due to checking more logical conditions resulting in less efficient system performance. Considering this as a key point, the combined balanced ternary number system is developed which works with this overflow technique in order to increase the system performance. In the combined balanced ternary number system, the continuous remainder testing and overflow technique is replaced by partial overflow technique. In the partial overflow technique the overflow during conversion for any specific value is prohibited. In this number system overflow value is introduced with the remainder rather than the convertible value which results in less number of condition checking during the entire conversion process. As less logical condition is used, this new number system sharpens the performance of the conversion.
\end{abstract}

Keywords:Binary number system, Ternary number system, Balanced ternary number system, SETUN, Combined balanced ternary number system, Decoding complexity.

\section{INTRODUCTION}

For decades we have been using binary number system as the core computation number system. Binary is the core due to its own easy and established logical theorem and set of properties in Boolean algebra. Another key reason behind using binary is its simplicity in representation, computation, usability and analysis. It goes beyond saying that the feasibility of designing binary components played the most vital role for the development and stability of binary number system. But demand has changed since then and we need more advance technology, increased speed, performance and efficiency.

Earlier in the first decade of computer invention, it was really tough to build a binary based computer due to lack of technology and horrible sized components, let alone higher based computer. But over time these two main problems were overcome which resulted in highly improved and efficient technology with reduced size. Then for the first time in history, Soviet Union dreamt of something big and started an experimental approach with the invention of first three based computer named "Setun". They found that the ternary system (base 3) was really complex to implement then. So, they went to the alternative way [6] to implement ternary and that was balanced ternary [7]. Later some actions were taken to invent a full-fill ternary based computer which is very shortly known. Now in the age of nanotechnology and top level electronics, there's a possibility of designing complete higher base computer than the binary computer and the first stepping stone is ternary.The design and implementation of ternary circuitry were reported in [11][12]. A new type of transmission functions theory was reported in [14]. Here, the author has suggested that this theory can explain all the CMOS ternary circuits.

Though the first step is to be made in ternary, the system is going to be unveiled through balanced ternary [1]. Ternary, more precisely balanced ternary is more suitable for improved and advance computing is suggested by author in [9]. The reason behind using the balanced ternary (though it is a nonstandard positional number system) is the unavailability of implementable standard ternary logics [2] whereas balanced ternary uses the advanced Boolean theorem developed from binary system. Also it was quite strenuous to represent ternary directly in computer due to technological bindings. Balanced ternary was far much easier [3] to implement rather than ternary and the prime cachet was the uniformity of digits in balanced ternary $(-1,1$, $0)$ respecting binary $(0,1)$. But the ultimate factor that energized to give priority to balanced ternary over ternary was its overflow method which resulted in a more forcible way with less complexity [5] and this overflow is the most crucial issue on the article. And in some cases it would be crucial also on cryptosystem [4]. Another fact is that Rotation Symmetric Boolean function has beckoned the interest of theoretician as well as practitioners in the field of cryptography [8] [10]. 
As mentioned earlier, the balanced ternary number system includes continuous overflow process which makes it effective to implement but results in a large number of logic checking. The combined balanced ternary is developed hereby to deal with this extra number of logic checking and continuous overflow.

\section{MATERIAL AND METHOD}

In this section, we describe the used techniques in computation (eg. Binary number system, balanced ternary number system) and the newly proposed combined balanced ternary number system. We have also put a comparison of some basic values of the number systems in tabular form.

In the early days of computing, a few experimental Soviet computers were built with balanced ternary instead of binary, the most famous being the Setun, built by Nikolay Brusentsov and Sergei Sobolev. The notation has a number of computational advantages over regular binary. Particularly, the plus-minus consistency cuts down the carry rate in multi-digit multiplication, and the roundingtruncation equivalence cuts down the carry rate in rounding on fractions. Balanced ternary also has a number of computational advantages over traditional ternary. And the new system that is developed on current time named "The Combined Balanced Ternary" is likely to replace the balanced ternary with more efficiency in performance.

\section{A. Existed Number Systems Analysis in Computation Scenario:}

There are numerous number systems. Yet we use the easiest possible number system with lower base for computation which gives us the advantage to do calculation in easier but long process. The basic number systems are Decimal, Binary, Octal, Hexadecimal etc. Among all the number systems, Binary is the simplest though it's not optimal. The optimal number system is " $\mathrm{e}$ " based. The nearest number system to optimal value is ternary number system that's why it is much significant.

Smaller the base, higher the bits to represent a value; this is the basic theory in computation. Still binary is used to develop computer system due to the simplicity and availability of theorems based on it. But when it comes in terms to ternary, we can't implement it in direct basis. Instead, we implement it in the nonstandard balanced ternary process which is closely connected to binary. Again we can't use high level based systems in large manner due to high electronics complexity. But, as it is important to upgrade the number system which is capable to provide better performance, the system should be initiated with the complete implementation of ternary number system. The proposed system below can be the initial step to do so.

Currently balanced ternary number system is used as the core to represent the ternary number system on the way to its implementation in computer system. But a major fact is that it is unable to represent the actual ternary number system. Perhaps, it is a binary converted form of ternary with continuous overflow technique. So, if we move towards the ternary representation, the first approach would be to deal with this continuous overflow technique. On the work we are focused with this fact.

\section{Traditional Ternary Number System:}

In traditional ternary the base value is 3 and the used digits are 0,1 and 2 . In digital system this two is currently unavailable to represent in direct independent bit form due to the binary logic properties of electronics. Let's take a look to a small conversion-

$(17)_{10}=(\ldots ? \ldots)_{3}$

Now, $\quad 3 / 17$

$3 / 1-2$

So, the conversion is: $(17)_{10}=(122)_{3}$

Reversely, $(122)_{3}=1 * 3^{2}+2 * 3^{1}+2 * 3^{0}$

$$
=9+6+1
$$

$=17$

\section{Balanced Ternary Number System:}

Balanced ternary number system is a nonstandard ternary representation form in binary properties. For a system it is found that the highest value is supposed to be 1 less than the base. But in balanced ternary, we can use a different system in conversion from any other type. Here we can introduce the 'overlap' technique. We jump on the next number if the remainder is only equal to 2 . And for that overlapping we will write the " -1 " in the place of remainder. Its advantage over the traditional ternary number system is that it is representable through binary system though it uses continuous overflow. Let's check a conversion-

$3 / 17$

$$
\text { 3/6-(-1) }
$$

$$
3 / 1-(-1)
$$

So, now the reverse conversion is:

$$
\begin{aligned}
& (17)_{10}=(1(-1) 0(-1))_{3} \\
& =1 * 3^{3}+(-1)^{*} 3^{2}+0 * 3^{1}+(-1) * 3^{0} \\
& =27+(-9)+0+(-1) \\
& =27-10
\end{aligned}
$$

$=17$ 


\section{B. Proposed System (Combined balanced ternary):}

In this section we are going to describe our proposed number system technique. Firstly, we would like to mention that the basic theme of this proposed system is influenced by the combination of balanced ternary and raw ternary, that's why we named it combined balanced ternary. Like the balanced ternary, it is a nonstandard number system but the proposed system differs from the balanced ternary in the sense of continuous overflow; in terms it uses a new method named partial overflow.

When it comes to continuous overflow, the value is randomly checked with its default criteria if overflow is possible or not. A worth mentioning factor about continuous overflow technique is that it is firstly checked through the remainder value and then executed on the root value.This lags the system through logical execution. Because the repeat of the overflow logic check in every times as well as new execution (more precisely computation) on the root value cost times which results in low performance.

If this repeat process of overflow possibility checking can be reduced then we are optimistic of getting better performance

On the contrary, the proposed system is based in partial overflow technique. In partial overflow, the overflow possibility is checked through the remainder but no extra computation is done over the root value, instead it deals with the remainder with the default value that is predetermined. This predetermined default value enables less logical execution than the continuous overflow technique.

\section{Predetermination of default value for partial overflow:}

The main problem we face in implementing ternary is due to its $3^{\text {rd }}$ value " 2 ." To represent this " 2 ", balanced ternary was developed. This value is responsible for the continuous overflow technique. So, if we want to do partial overflow technique, we need to deal with this value. The only overflow we will use in our partial overflow technique is associated with this value "2." Let's take overflow for 2 -

bar as only 1 value is elapsed. Thus the positional value of "P" would be $3^{\mathrm{n}+1}$ which is similar to the last value " 1 " under the bar. This relationship can also be stated as that the positional value of the digit next to the bar would be equal to the adjacent bar digit. On the example "P" and 1 (adjacent digit of bar) would have the same positional value. This would be the same for all similar situation.

\section{Final conversion:}

In, this section, we will show the execution of the proposed system. For this, we will take any random number for applying our technique. Let take 33. Now, we will convert this value in our proposed "Combined balanced ternary number system" and the will reverse it back to the decimal value.

$$
3 / 2=1(-1)
$$

So, $(2)_{10}=(1(-1))_{3}$

Now reversely, $1 * 3^{1}+(-1) * 3^{0}=3-1=2$. Thus the overflow balanced ternary value for 2 is $1(-1)$. But in our partial overflow technique we will represent 2 by $\overline{\mathbf{1}(-\mathbf{1})}$ instead of 1(-1). The bar over 1(-1) means the entire digits under the bar would be represented as one digit.

\section{Substitution between remainder and overflow value:}

Substitution between the remainder and pre-determined value is a pivotal factor in our partial overflow technique. During ternary conversion, we will use no overflow in the system. Instead of continuous overflow we will only substitute bits.

The substitution will be done with the remainder only when the remainder becomes " 2 ." If the remainder is " 2 " it will be replaced by the pre-determined default value $\overline{\mathbf{1 ( - 1 )}}$. This substitution keeps the root value free from continuous overflow in conversion.

\section{Positional value assigning during reversing:}

As we used $\overline{\mathbf{1}(\mathbf{- 1})}$ instead of $1(-1)$ and the entire digits under the bar appeared to be a single digit, we need to make a change in assigning the positional value. In any sequence that contain $\overline{\mathbf{1}(-\mathbf{1})}$, the right $(-1)$ under the bar would follow the sequence of positional power and so as the next 1. But when the computation will complete the bar it would count as only one positional power has elapsed. Let's consider a case for better understanding-

Suppose, "P $\overline{\mathbf{1}(-\mathbf{1})} \mathrm{Q}$ " is a sequence which need to be reversed. Now, let's consider the positional power of " $Q$ " is $3^{\mathrm{n}-1}$ then the positional power of $(-1)$ under the bar would be $3^{n}$, and for 1 under the bar it would be $3^{n+1}$. But when the process will be executed right after the bar, it will calculate the

whole

$$
\begin{aligned}
& 3 / 33 \\
& 3 / 11-0 \\
& 3 / 3-2=[3-(\overline{\mathbf{1}(-\mathbf{1})})] \\
& \quad 3 / 1-0
\end{aligned}
$$

So, the converted value in combined balanced ternary would be, $\quad[10 \overline{\mathbf{1}(-\mathbf{1})} 0]_{3}$.

Reversely, $[10 \overline{\mathbf{1 ( - 1 )}} 0]_{3}=1 * 3^{3}+0 * 3^{2}+1 * 3^{2}+(-1) * 3^{1}+0 * 3^{0}$

$=27+0+9-3+0=33$

Thus, $(33)_{10=}[10 \overline{\mathbf{1}(-\mathbf{1})} 0]_{3}$

This conversion process is true for any value "n."

\section{Algorithm and flowchart:}

Algorithm for "Combined balanced ternary" conversion from decimal value is given below-

$$
(33)_{10}=(. . ? \ldots)
$$


Step 1. Initialize the value $(\mathrm{X})$ to be converted in ternary and select $\mathrm{P}=3$.

Step 2. Do steps 3 to 5 when $X \geq 3$.

Step 3. Divide $\mathrm{X}$ by $\mathrm{P}$ and keep the remainder $(\mathrm{R})$ sequentially.

Step 4. If $\mathrm{R}=2$ go to step 5, else continue.
Step 5. Replace R by $\overline{\mathbf{1}(-\mathbf{1})}$ and keep sequentially.

Step 6. If $X=2$ go to step 7 else keep the value as remainder.

Step 7. Replace X by $\overline{\mathbf{1}(-\mathbf{1})}$ and keep sequentially.

Step 8. Stop.

The flowchart is given below-

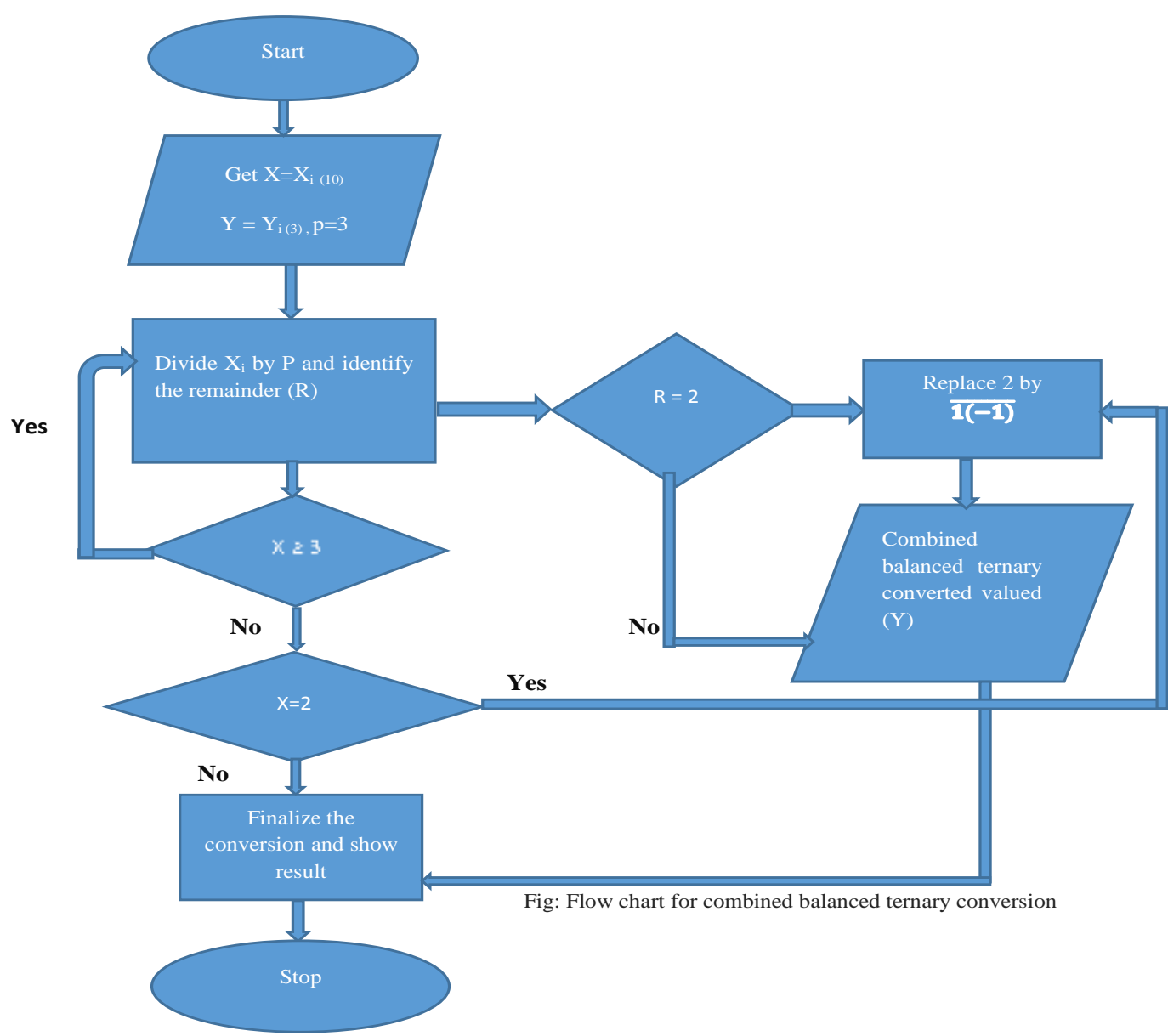

\section{RESULT AND DISCUSSION}

In this section we will discuss about the outcome from the proposed number system and its potentiality to the computation. The primary goal of this work was to develop a system that would be more precise to ternary system than the balanced ternary system.

\section{A. Outcomes:}

Thecombined balanced ternary number system can be said as the sister number system of both balanced ternary and ternary. It has a completely new technique of ternary conversion. The core outcomes of the proposed system are as follows-

\section{Creation of a new number system:}

Binary number system is the core number system of computer currently. Ternary number system is the future but it isn't implementable directly thus balanced ternary is developed. ON the sequence, the combined balanced ternary is another addition as an implementable ternary number system form through binary. This number system is unique in its own technique. And, of course it's worth mentioning that the partial over flow technique used in the system is totally new in the era of mathematics and 
number system. This technique will open some new ways to rebuild or reshape any systems through allowing to think in a different manner with the number systems. A major factor is that it will insist to consider the existed number system to be reconstructed and there may rise some new potentiality with the rebuilt systems.

\section{Lessening the complexity of the balanced ternary:}

The basic goal of this new number system is to reduce the complexity of the implementation of balanced ternary though it is in the initial development process. The newly developed combined balanced ternary number system is expected to replace the balanced ternary number system over time due to some potential computational mathematical properties such as-

Firstly,less use of logic than the balanced ternary number system. Still it works explicitly like the balanced ternary.

Secondly,it is expected to provide all the past benefits of balanced ternary number system though it requires complete development of the system.

\section{Increase the speed of computation:}

Another prime outcome of this proposed system is to achieve higher rate of throughput in the computation work. And this can be simply achieved through using the newly introduced number system as the processor (Yet to develop any) of this number system would be simpler than the processor of balanced ternary due to lessening the number of logic that was needed to be executed in the previous system.

\section{B. Comparative value table:}

Among all the number systems, four number systems are most widely used. They are Decimal, Octal, Hexadecimal and Binary. As we are intended to discuss about ternary, we have given a value table that includes the comparative value between the systems as well as including the Balanced ternary and proposed Combined balanced ternary system.Here is a combined ternary value table comparing to other systems:

\begin{tabular}{|c|c|c|c|c|c|c|}
\hline Decimal & Hexadecimal & Octal & Binary & Ternary & Balanced Ternary & Combined Balanced Ternary \\
\hline 0 & 0 & 0 & 0 & 0 & 0 & 0 \\
\hline 1 & 1 & 1 & 1 & 1 & 1 & 1 \\
\hline 2 & 2 & 2 & 10 & 2 & $1(-1)$ & $\overline{1(-1)}$ \\
\hline 3 & 3 & 3 & 11 & 10 & 10 & 10 \\
\hline 4 & 4 & 4 & 100 & 11 & 11 & 11 \\
\hline 5 & 5 & 5 & 101 & 12 & $1(-1)(-1)$ & $1 \overline{1(-1)}$ \\
\hline 6 & 6 & 6 & 110 & 20 & $1(-1) 0$ & $\overline{1(-1)} 0$ \\
\hline 7 & 7 & 7 & 111 & 21 & $1(-1) 1$ & $\overline{1(-1)} 1$ \\
\hline 8 & 8 & 10 & 1000 & 22 & $10(-1)$ & $\overline{1(-1)} \overline{1(-1)}$ \\
\hline 9 & 9 & 11 & 1001 & 100 & 100 & 100 \\
\hline 10 & A & 12 & 1010 & 101 & 101 & 101 \\
\hline 11 & B & 13 & 1011 & 102 & $11(-1)$ & $10 \overline{1(-1)}$ \\
\hline 12 & $\mathrm{C}$ & 14 & 1100 & 110 & 110 & 110 \\
\hline 13 & $\mathrm{D}$ & 15 & 1101 & 111 & 111 & 111 \\
\hline 14 & E & 16 & 1110 & 112 & $1(-1)(-1)(-1)$ & $11 \overline{1(-1)}$ \\
\hline 15 & $\mathrm{~F}$ & 17 & 1111 & 120 & $1(-1)(-1) 0$ & $1 \overline{1(-1)} 0$ \\
\hline
\end{tabular}


C. Limitations:Nothing is free of limitation. There's a few limitations in newly developed combined balanced ternary though it is in the initial development process. The major limitations are stated below:

$i$.The main limitation of the system is its decoding complexity due to its totally new concept of positional value.

ii. Another key limitation is that it requires higher number of memory space than the previously used systems in some cases.

\section{CONCLUSIONS}

It is beyond saying that the ternary number system is the upcoming future of computational mathematics. Though it is expected that the balanced ternary system will rule in ternary computing, the newly introduced combined balanced ternary system would be a strong contender to balanced ternary in implementation if the new system is developed properly with full characteristics.

The development of the combined balanced ternary is currently on its initial process. There's still a lot things to do in order to develop a complete combined balanced ternary number system for implementation purpose. Once the system is developed and it is in its full form then it could be an epoch making move in the era of computation sector.

\section{REFERENCES}

1. Third Base by Brian Hayes, American scientist, Vol. 89, No.6, 2001, pp. 490-494.

2. A New Moduli Set for Residue Number System in Ternary Valued Logic by M. Hosseinzadeh and K. Navi.[Research paper published on Science Alert].

3. Constructions of balanced ternary designs by D. G. Sarvate [Cambridge University Press,Journal of the Australian Mathematical Society]

4. J. Adikari, V. S. Dimitrov, L. Imbert, Hybrid Binary-Ternary Number System for Elliptic Curve Crypto System, IEEE Transactions on Computers, Vol-60, No.-2, Feb-2011.
5. Towards a balanced ternary FPGA by Paul Beckett Electrical \& Computer Engineering, RMIT University, Latrobe St., Melbourne, Australia.

6. : D. Donovan, 'Methods for constructing balanced ternary designs',Ars Combin. 26A, to appear.

7. A. Francel, Margaret \& Hurd, Spencer. (2008). Nested balanced ternary designs and Bhaskar Rao designs. The Australasian Journal of Combinatorics [electronic only].

8. P Stanica and S Maitra, Rotation Symmetric Boolean Functions-Count and Cryptographic Properties, Discrete Applied Mathematics,Vol156,n0.-10,May 2008.

9. Balanced- Ternary Logic for Improved and Advanced Computing by Shamshad Ahmad, Mansaf Alam Deptt. Computer Science, JMI, New Delhi 10.

10. 10. P. Sarkar, S. Maitra, Constructions of Nonlinear Boolean Functions with Important Cryptographic Properties. In Advances is CryptologyEUROCRYPT 2000,pp 485-506,Springer Verlog, 2000.

11. A, Srivastava, K Venkatapathy," Design and implementation of a low power ternary full adder", VLSI Design,1996,VOl-4, No.-1,pp 75-81

12. A.Sathish Kumar, A. Swetha Priya, The Minimization of Ternary Combinational Circuits -A Survey,A. Sathish Kumar et.al./International Journal of Engineering and Technology,Vol2(8),2010,pp 35376-3589,ISSN 0975-5462.

13. A.P.Dhande, V.T. Ingole, Design and Implementation of 2 Bit ternary ALU Slice, Third International Conference; SETIT 2005-Tunisia.

14. X.W.Wu, CMOS Ternary Logic Circuits, IEEE Proceedings, Vol 137, Pt.G, No. 1, Feb 199 